\title{
Jamaica: tamaño de las empresas y remuneración laboral en el sector privado
}

\author{
Allister Mounsey
}

RESUMEN

\begin{abstract}
Los datos sugieren que los mercados laborales no encuentran el equilibrio según la microeconomía tradicional. Las persistentes desigualdades salariales intersectoriales y aquellas derivadas del tamaño de la empresa constituyen un desafío. Los datos del sector privado de Jamaica revelan que las desigualdades salariales según tamaño de la empresa podrían promover desigualdades intersectoriales. Al considerar la calidad de la mano de obra y otras características, se descubre que las empresas con 10 a 49 empleados y aquellas con 50 o más pagan primas estimadas de $14,3 \%$ y $22,9 \%$, respectivamente. Tras estimar las diferencias en los perfiles de permanencia laboral, la prima vinculada a empresas mayores se redujo al 15,9\%, y no varió la vinculada a empresas con 10 a 49 trabajadores. No obstante la explicación parcial proporcionada por los diferentes perfiles de permanencia laboral, el grueso de las desigualdades salariales según tamaño de la empresa parece obedecer a otras construcciones teóricas.
\end{abstract}




\section{I}

\section{Introducción}

La existencia de relaciones positivas entre el número de trabajadores de una empresa y los salarios que reciben, es decir, la presencia de desigualdades salariales según el tamaño de la empresa, constituye una característica del mercado laboral bien documentada en las economías avanzadas. Lester (1967) fue uno de los primeros investigadores que documentaron de forma exhaustiva la existencia de dichas desigualdades en los Estados Unidos de América. Descubrió que las empresas con 2.500 trabajadores o más tenían un nivel combinado de salarios y prestaciones que era al menos un $20 \%$ más alto, en promedio, que el de las empresas con entre 20 y 100 empleados. Masters (1969) demostró que las desigualdades salariales según el tamaño de la empresa siguen existiendo después de haber tenido en cuenta la influencia de la competencia en el mercado y la sindicalización. Otros autores confirman este fenómeno en los Estados Unidos de América (Antos, 1983; Mellow, 1982; Oi, 1983). Winter-Ebmer y Zweimüller (1999) proporcionan datos del mismo fenómeno en las pequeñas y medianas empresas de Suiza, mientras que Fakhfakh y Fitzroy (2006); Oosterbeek y van Praag (1995), y Main y Reilly (1993) encuentran datos similares en Francia, los Países Bajos y el Reino Unido de Gran Bretaña e Irlanda del Norte, respectivamente.

Resulta igualmente desconcertante la existencia de desigualdades salariales intersectoriales que no varían con el tiempo. Se considera que Slichter (1950, citado en Krueger y Summers, 1987) fue el primero en observar este fenómeno. Slichter encontró un coeficiente intertemporal de correlación de rangos de 0,73 en los salarios de 20 sectores manufactureros de los Estados Unidos de América entre 1923 y 1946. Se han documentado resultados similares tanto en este último país como internacionalmente (por ejemplo, Krueger y Summers, 1987; Gittleman y Wolff, 1993; Arbache, 2001). El hecho de que las desigualdades salariales según el tamaño de la empresa y las desigualdades salariales intersectoriales estén muy generalizadas pone en duda la suposición fundamental neoclásica de la existencia de salarios de equilibrio. Las desigualdades salariales según el tamaño de la empresa, en particular, constituyen un desafío significativo para la teoría tradicional de la empresa, la que — según Lester (1967) — no puede explicar por qué los empleadores con mayor poder monopolístico ofrecen generalmente los niveles más altos de salarios y prestaciones. Muchos autores consideran que aún no hay respuesta para estas dificultades de la comprensión tradicional de los mercados laborales y de las actividades empresariales, a pesar de los intentos por explicarlas en el marco de maximización competitiva de beneficios de la economía neoclásica.

Los niveles de desempleo persistentemente elevados en el Caribe subrayan la necesidad de investigar estos fenómenos en la región ${ }^{1}$. Si bien las desigualdades salariales intersectoriales han recibido cierta atención en el Caribe de habla inglesa (Mounsey y Polius, 2011), el fenómeno de las desigualdades salariales según el tamaño de la empresa no ha sido investigado. En este estudio se intenta proporcionar una base que permita investigar el tema con más profundidad, presentando datos que evidencian la existencia de desigualdades salariales según el tamaño de la empresa y de desigualdades salariales intersectoriales en Jamaica. Se demuestra que las desigualdades salariales intersectoriales observadas podrían explicarse, en principio, en función de la proporción del total de la fuerza laboral del sector empleada por las empresas grandes o pequeñas, y se plantea la posibilidad de que las desigualdades salariales según el tamaño de la empresa sean la causa de las desigualdades salariales intersectoriales ${ }^{2}$. También se estudia en qué medida son adecuadas las explicaciones de las desigualdades salariales según el tamaño de la empresa basadas en la calidad laboral y en las diferencias en los perfiles de permanencia en el empleo.

El resto del estudio se divide en cinco secciones. En la sección II se revisan algunas de las explicaciones teóricas de las desigualdades salariales según el tamaño de la empresa. Los datos utilizados se indican en la sección siguiente. En la sección III se demuestra la relación existente entre las desigualdades salariales según el tamaño de la empresa y las desigualdades salariales intersectoriales. La estimación y los resultados se presentan en la sección IV y las conclusiones del estudio en la sección V.

\footnotetext{
${ }^{1}$ Los datos de la Organización Internacional del Trabajo (OIT) revelan que la tasa de desempleo anual media en Barbados, Jamaica y Trinidad y Tabago era del $8,6 \%, 10,8 \%$ y $6,5 \%$, respectivamente, en el período comprendido entre 2004 y 2008 (países seleccionados por razones de disponibilidad de los datos).

2 En este estudio no se examina la estabilidad temporal de las desigualdades salariales intersectoriales ni de las desigualdades salariales según el tamaño de la empresa en Jamaica, debido a limitaciones temporales de los datos utilizados.
} 


\section{II}

\section{Explicaciones teóricas de las desigualdades salariales según el tamaño de la empresa}

Las explicaciones de las desigualdades salariales según el tamaño de la empresa pueden incluirse en dos categorías: las explicaciones competitivas y las no competitivas. Según estas últimas, las características institucionales endógenas de las empresas de cada tamaño particular dan lugar a remuneraciones diferentes para trabajadores con capacidades y experiencia comparables, según el tamaño de la empresa. En el caso de las primeras explicaciones, se considera que las empresas de distinto tamaño ofrecen condiciones laborales diferentes o requieren diversos niveles de calidad de la mano de obra. Los trabajadores deben ser compensados por estas diferencias (de condiciones laborales o de calidad de la mano de obra) y se producen las desigualdades salariales observadas, dependiendo del tamaño de la empresa.

\section{Explicaciones competitivas: diferencias compensatorias}

El resumen paradigmático de la teoría de las diferencias compensatorias se encuentra en Smith (1904), quien en 1776 afirmaba que el salario del trabajador varía con la facilidad o la dificultad, la limpieza o la suciedad, y lo honroso o deshonroso del trabajo. En la teoría de las diferencias compensatorias, por lo tanto, se sostiene que las desigualdades salariales según el tamaño de la empresa existen a causa de las desigualdades en las condiciones laborales o en la calidad de la mano de obra.

Las explicaciones basadas en las condiciones laborales, que no se analizan en este estudio debido a limitaciones relacionadas con los datos, se centran en los aspectos menos atractivos de trabajar en grandes empresas, como un entorno centrado en las normas y la falta de control sobre el horario y las propias actividades (Masters, 1969; Duncan y Stafford, 1980). En la explicación basada en la calidad de la mano de obra se afirma que las empresas de mayor tamaño buscan de manera activa mano de obra de más calidad, en comparación con otras empresas. Una posible motivación para esta forma de actuar consiste en la tendencia por parte de las empresas de mayor tamaño a utilizar tecnologías más intensivas en capital y en el hecho de que la mano de obra altamente calificada y el capital son complementarios (Hamermesh y Grant, 1979). Por consiguiente, la complementariedad entre el capital y la calificación de la mano de obra hace que las empresas de mayor tamaño y más intensivas en capital empleen a trabajadores de mayor calificación, que reciben un salario superior determinado por el mercado.

Rosen (1982) propuso una motivación diferente, que no se basa en la calificación del trabajador típico, sino más bien en la calificación de las posiciones de alta dirección de las empresas de mayor tamaño, en comparación con las de menor tamaño. Rosen sostiene que el aumento de productividad resultante del nombramiento de personas con una calificación superior para que desempeñen posiciones de alta dirección es mayor que el incremento de sus capacidades, debido a los filtros de calificación a lo largo de toda la cadena de mando inferior. En una situación de este tipo, el mercado competitivo de gerentes y supervisores se equilibrará incidiendo en que los puestos de alta dirección de las grandes empresas sean desempeñados por personas con un elevado nivel de calificación, quienes recibirán una mayor remuneración, mientras que los puestos más altos de las empresas de menor tamaño son desempeñados por personas con menor calificación y con una menor remuneración.

En la hipótesis del costo de monitoreo de Oi (1983) se sugiere otra posible motivación. Los empresarios tienen idéntica capacidad para monitorear a los trabajadores, pero distinta capacidad para coordinar la producción. Por lo tanto, la limitación del tiempo disponible da lugar a un costo sombra relativamente alto vinculado al monitoreo por parte de los empresarios con más calificación, que suelen dirigir empresas de mayor tamaño. Los empresarios de grandes empresas intentan minimizar el elevado costo sombra de monitorear a los trabajadores contratando mano de obra de mayor calidad, que es más productiva, necesita un menor grado de monitoreo por unidad de eficiencia y requiere una mayor remuneración determinada por el mercado, debido a su calidad más alta.

Quienes defienden las explicaciones de las desigualdades salariales según el tamaño de la empresa basadas en la calidad de la mano de obra señalan que esa calidad está formada por características medidas, como la educación y la experiencia, y características no medidas. Las primeras se comprueban fácilmente utilizando series de datos transversales fácilmente accesibles, mientras 
que comprobar la importancia de la calidad no medida suele requerir datos longitudinales más costosos y menos accesibles (Brown y Medoff, 1989).

\section{Explicaciones no competitivas}

Existen varias explicaciones no competitivas de las desigualdades salariales según el tamaño de la empresa. A grandes rasgos, pueden dividirse en las que son coherentes con la suposición de que las empresas maximizan los beneficios (y minimizan los costos) y las que no lo son. Entre estas últimas, probablemente la más conocida sea la hipótesis de poder de mercado del producto, en que se afirma que las empresas con poder monopolístico (es decir, aquellas de mayor tamaño) utilizan parte de su exceso de beneficios para compartir en alguna medida sus ingresos con sus trabajadores (Brown y Medoff, 1989). Uno de los problemas principales de la hipótesis de poder de mercado del producto es su incapacidad para explicar por qué la competencia por estos empleos mejores no da lugar a una fuerza de trabajo con mayor calificación, pero sin una mayor remuneración (Brown y Medoff, 1989).

Entre las explicaciones coherentes con la maximización de beneficios, se encuentran los modelos de salariales de eficiencia y las explicaciones basadas en las diferencias en los perfiles salariales y de permanencia en el empleo según el tamaño de las empresas. A lo largo del resto de esta subsección, se proporcionan breves resúmenes de cada una de estas explicaciones no competitivas.

Riveros y Bouton (1994) definen los modelos de salariales de eficiencia como una familia de teorías conceptualmente distintas con las que, en su mayor parte, se intenta ofrecer una explicación (endógenamente determinada) de las persistentes rigideces de los salarios reales en presencia de desempleo involuntario. La suposición fundamental de estas teorías es que unos salarios reales más altos pueden dar lugar a una mayor productividad laboral, a través de diversos mecanismos. Hay tres principales modelos de salariales de eficiencia: i) el modelo de monitoreo e incumplimiento de los deberes; ii) el modelo de costo de rotación del personal, y iii) el modelo sociológico. A continuación se describen cada uno de estos modelos (adaptado de Mounsey y Polius, 2011).

i) Shapiro y Stiglitz (1984) demostraron que, en condiciones de monitoreo imperfecto, el paradigma competitivo básico de la economía neoclásica alcanza el equilibrio en el punto en que el incumplimiento de los propios deberes es común a todos los trabajadores. Para lograr que los trabajadores se esfuercen más, las empresas pagan salarios por sobre el salario de equilibrio, creando así una penalización para los empleados que no cumplen sus deberes y son despedidos ${ }^{3}$. Bulow y Summers (1986) ampliaron el marco básico de Shapiro y Stiglitz para mostrar cómo trabajadores igualmente productivos pueden ser asignados arbitrariamente, en el punto de equilibrio, a un sector con salarios altos o a uno con salarios bajos, de manera que las empresas con salarios elevados son aquellas para las que el incumplimiento de los empleados de sus deberes resulta más costoso o en las que es más difícil monitorear la conducta de los empleados. En relación con el tamaño de las empresas, los defensores de este modelo afirman que el monitoreo es más difícil y probablemente el incumplimiento de deberes resulta más costoso en las empresas de mayor tamaño.

ii) El modelo de costo de rotación del personal plantea que el abandono de la empresa por los empleados hace que el empleador sufra costos irrecuperables vinculados a la contratación y formación de nuevos trabajadores, así como una pérdida de productividad mientras esos nuevos empleados avanzan a lo largo de la curva de aprendizaje. En las empresas se intenta minimizar estos costos de rotación del personal pagando un salario más alto, para fomentar la permanencia de los trabajadores (Salop, 1979). Los defensores de este modelo afirman que, para cualquier empleo concreto, puede haber una relación positiva entre los costos de rotación de personal y el tamaño de la empresa, ya que las empresas de mayor tamaño suelen utilizar técnicas de producción más especializadas y, por lo tanto, invierten más en la formación de cada trabajador. En consecuencia, la distribución de salarios también se relaciona positivamente con el tamaño de la empresa.

iii) Akerlof (1982 y 1984) sostiene que las costumbres o normas sociales en el entorno laboral tienen un fuerte efecto en la actitud de los trabajadores. Los empleados se ven motivados a trabajar duro por un sentimiento de fidelidad con respecto a los demás empleados y a la empresa. A cambio de su compromiso, los trabajadores esperan recibir un salario justo. Este salario justo depende del grupo salarial de referencia y de los salarios anteriores, entre otras cosas. Según el modelo sociológico básico, la lealtad de los empleados recibe como contrapartida un salario mayor y esta lealtad puede transformarse, por medio de una administración eficaz, en un alto nivel de productividad (Akerlof, 1984, pág. 80). Las desigualdades salariales entre distintas empresas (o

\footnotetext{
3 La amplitud de la desigualdad salarial depende del costo que suponen para la empresa los empleados que no cumplen sus deberes.
} 
distintos sectores) pueden deberse a la diversa capacidad de las empresas (o sectores) para transformar la fidelidad de sus empleados en una mayor productividad.

Otra explicación de la relación positiva entre los salarios y el tamaño de las empresas se basa en las diferencias en el perfil de permanencia en el empleo de las grandes empresas, en comparación con las pequeñas. Según esta explicación, las grandes empresas invierten más en formación específica del sector que las pequeñas empresas. De este modo, las grandes empresas manifiestan un especial interés en conservar a sus empleados. Una manera de conservarlos consiste en el uso de las primas por antigüedad, gracias a las cuales el salario del trabajador es mayor cuando ha trabajado más años para la empresa (Lazear, 1979 y 1981). De esta forma, se crea un perfil salarial de permanencia en el empleo y se introduce un elemento de compensación diferida, casi equivalente a que el trabajador deposite una fianza en la empresa. Por lo tanto, se modifica la estructura de incentivos a los empleados estimulando el trabajo duro y la honradez, como formas de alcanzar finalmente el período de reembolso del depósito (Zwick, 2009) ${ }^{4}$. En consecuencia, el interés de las grandes empresas da lugar a perfiles más altos de permanencia en el empleo en firmas de mayor tamaño y podría explicar las desigualdades salariales según el tamaño de la empresa.

\section{Datos del estudio}

La fuente de los datos utilizados en este estudio corresponde a la Encuesta sobre la Fuerza de Trabajo de Jamaica (JLFS por sus siglas en inglés), que es realizada trimestralmente por el Instituto de Estadística de Jamaica (STATIN por sus siglas en inglés). Se utiliza la encuesta del segundo trimestre de cada año desde 2004 hasta 2007. Las variables seleccionadas corresponden a participantes en la encuesta que afirmaban haber trabajado en el sector privado durante el período de referencia de cada encuesta 5 . Hubo 7.667 participantes con esta característica, es decir, en torno del 74,7\% de los encuestados que declararon tener empleo. Véase en el cuadro 1 un desglose de la muestra a lo largo del período considerado ${ }^{6}$.

4 La existencia de perfiles de permanencia en el empleo también puede estar motivada por una perspectiva de monitoreo (véanse Pearce, 1990; Zwick, 2009).

5 También se excluyeron los trabajadores por cuenta propia, los trabajadores familiares no remunerados y los empleadores.

6 La elección del segundo trimestre fue totalmente arbitraria; sin embargo, se seleccionó el mismo trimestre cada año, en lugar de cuatro trimestres en el mismo año, por ejemplo, para mitigar el impacto de la estacionalidad en los regresores.
CUADRO 1

\section{Desglose temporal de la muestra}

\begin{tabular}{lccccc}
\hline \multirow{2}{*}{ Muestra } & \multicolumn{4}{c}{ Período (segundo trimestre) } & \multirow{2}{*}{ Total } \\
\cline { 2 - 5 } & 2004 & 2005 & 2006 & 2007 & \\
\hline $\mathrm{N}^{\mathrm{o}}$ de encuestados & 2131 & 1765 & 2016 & 1755 & 7667 \\
\hline
\end{tabular}

Fuente: elaboración propia sobre la base de datos de la Encuesta sobre la Fuerza de Trabajo, segundo trimestre de los años 2004 a 2007, Instituto de Estadística de Jamaica (STATIN).

Las variables utilizadas en este estudio son el grupo ocupacional principal del trabajador (OCC), el grupo principal de sectores (IND), su permanencia o antigüedad en el trabajo actual (TEN), el logaritmo de los ingresos anuales brutos por el empleo principal (LAE), los años de experiencia (EXP), los años de educación efectiva (YS), el género (GEN) y las horas regulares de trabajo a la semana (HWKD). También se utiliza información sobre si el trabajador ha recibido capacitación formal en el empleo (OJT), si sus logros educativos le permiten al menos matricularse en estudios terciarios (TMAT) ${ }^{7}$, el tamaño de la empresa medido como el número de personas empleadas en el lugar de trabajo principal del trabajador $(E S)$ y si ese lugar de trabajo está situado en Kingston, en una zona rural o en otra zona urbana $(\mathrm{KRU})^{8}$.

Las variables $Y S, L A E$ y EXP se obtuvieron mediante las siguientes transformaciones de las variables de la Encuesta sobre la Fuerza de Trabajo de Jamaica

$$
Y S=\left\{\begin{array}{c}
16, \text { si Hcert }=\text { Título universitario } \\
13, \text { si Hcert }=\text { Pruebas de ingreso a la universidad } \\
P+S, \text { en los demás casos }
\end{array}\right.
$$

donde Hcert es la certificación más alta obtenida y $P$ y $S$ son años de educación primaria y secundaria, respectivamente.

7 La inclusión de la variable TMAT tiene por objeto realizar una distinción cualitativa dentro del grupo constituido por personas con 11 años de educación (educación secundaria finalizada), que integran el $46,2 \%$ de la muestra.

${ }^{8}$ Las variables en cursiva son aquellas que han sido transformadas a partir de variables originales. TEN es una variable con tres categorías, correspondientes a menos de dos años, entre dos y cinco años y más de cinco años en el trabajo actual. La variable original de la que se deriva TEN tiene siete categorías (cuatro de las cuales se refieren a personas que han trabajado para su empresa actual durante menos de un año). La variable Es incluía originalmente cinco categorías; pero, para mayor concisión, se redujo a tres categorías, uniendo las tres primeras. 


$$
E X P=A G E-Y S-5
$$

donde $A G E$ es el número de años de edad de la persona, que se obtiene de la Encuesta sobre la Fuerza de Trabajo de Jamaica

$$
L A E=\ln G A E
$$

donde GAE es la cifra de ingresos brutos anuales del trabajador obtenidos en su empleo principal, tal como se contabiliza en la Encuesta sobre la Fuerza de Trabajo de Jamaica9.

9 Los ingresos de cada participante se indicaron con tres posibles periodicidades (semanal, mensual o anual). Cuando los ingresos se
En el cuadro 2 se observa la distribución de participantes en la encuesta por sector y por tamaño de la empresa. En lo referente a los sectores, como cabe esperar de una economía orientada a los servicios, la muestra está muy concentrada en el sector de servicios comunitarios, sociales y personales, en el sector mayorista y minorista y de hoteles y restaurantes. En cuanto al tamaño de las empresas, más de la mitad de los trabajadores del sector privado corresponden a empresas con nueve empleados o menos, un dato que también es coherente con la orientación a los servicios de la economía del país.

indicaron con periodicidad semanal o mensual, se multiplicaron por 52 o por 12, respectivamente, para convertirlos en ingresos anuales.

CUADRO 2

Distribución de participantes en la encuesta por sector y tamaño de la empresa

\begin{tabular}{|c|c|c|c|c|}
\hline \multirow{2}{*}{ Grupo de sectores (un dígito) } & \multicolumn{3}{|c|}{$\begin{array}{l}\text { Tamaño de la empresa } \\
\text { (número de empleados) }\end{array}$} & \multirow{2}{*}{ Total } \\
\hline & $<10$ & $10-49$ & $\geq 50$ & \\
\hline Agricultura, ganadería, silvicultura y pesca (1) & 191 & 118 & 84 & 393 \\
\hline Construcción e instalaciones (2) & 683 & 281 & 53 & 1017 \\
\hline Servicios comunitarios, sociales y personales (3) & 1704 & 263 & 64 & 2031 \\
\hline Electricidad, gas y agua (4) & 2 & 12 & 17 & 31 \\
\hline Servicios financieros, de seguros, inmobiliarios y a las empresas (5) & 106 & 313 & 225 & 644 \\
\hline Minería, canteras y refinado (6) & 3 & 25 & 93 & 121 \\
\hline Manufacturas (7) & 182 & 295 & 224 & 701 \\
\hline Transporte, almacenamiento y comunicaciones (8) & 277 & 116 & 110 & 503 \\
\hline Mayorista, minorista, hoteles y restaurantes (9) & 748 & 782 & 342 & 1872 \\
\hline Total & 3896 & 2205 & 1212 & 7313 \\
\hline
\end{tabular}
(En número de participantes)

Fuente: elaboración propia sobre la base de datos de la Encuesta sobre la Fuerza de Trabajo, segundo trimestre de 2004 a 2007 , Instituto de Estadística de Jamaica (STATIN).

Nota: el tamaño de la empresa no se indicó en 354 respuestas. 


\section{III}

\section{Relación entre las desigualdades salariales según el tamaño de la empresa y las desigualdades salariales intersectoriales}

En esta sección se presenta una breve investigación estadística sobre las desigualdades salariales intersectoriales y las desigualdades salariales según el tamaño de la empresa. Se denomina $G A E_{i j k t}$ la cifra de ingresos anuales indicada por la persona $i$, en el empleo $j$, correspondiente al sector $k$, durante el año $t$. El salario por hora recibido por esa persona se estimará de forma aproximada, como se indica a continuación ${ }^{10}$ :

$$
w_{i j k t}=\frac{G A E_{i j k t}}{52(H W K D)} .
$$

Los datos sobre la ocupación se proporcionan mediante un código de cuatro dígitos. El salario medio por hora para cada código de ocupación de cuatro dígitos se calcula, respecto de cada año, utilizando la fórmula siguiente:

$$
\overline{w_{j k t}}=\frac{\sum_{\forall i i n t} w_{i j k t}}{n_{j t}},
$$

\footnotetext{
${ }^{10}$ En la serie de datos no hay información disponible sobre las semanas reales trabajadas al año, de manera que, para los efectos de estimar el salario por hora, se ha supuesto un número constante de semanas trabajadas (52 semanas al año).
}

donde $n_{j t}$ es el número de personas en la ocupación $j$, en el año $t$.

Finalmente, el salario relativo del trabajador ( $r w)$ (en relación con el salario medio recibido por la cohorte ocupacional) se calcula como se indica a continuación:

$$
r w_{i j k t}=w_{i j k t} / \frac{}{w_{j k t}} \forall i y t .
$$

En el cuadro 3 se aprecia la distribución del salario relativo medio $\left(\overline{r w_{k}}\right)$ por grupo de sectores y por tamaño de la empresa ${ }^{11}$. Un salario relativo medio $\left(\overline{r w_{k}}\right)$ inferior a uno (por ejemplo, igual a 0,75 ) significa que se puede esperar que un trabajador seleccionado al azar en el sector $k$ reciba un salario por hora un $25 \%$ inferior al

11 El salario relativo medio del sector $k$ se obtiene mediante el siguiente cálculo:

$$
\overline{r w_{k}}=\sum_{i} r w_{i j k t} / N_{k} \forall i \text { in } k
$$

donde $N_{k}$ es el número de elementos de la muestra en el sector $k$. Se utiliza un cálculo similar para obtener el salario relativo medio según el tamaño de la empresa en el grupo de sectores $k$.

CUADRO 3

Salario relativo medio $\left(\overline{r w_{k}}\right)$ por grupos de sectores y tamaño de la empresa

\begin{tabular}{lcccc}
\hline & & \multicolumn{2}{c}{$\begin{array}{c}\text { Tamaño de la empresa } \\
\text { (número de empleados) }\end{array}$} & $\begin{array}{c}\text { Todas las } \\
\text { empresas }\end{array}$ \\
\cline { 2 - 4 } & $<10$ & $10-49$ & $\geq 50$ & 0,973 \\
Agricultura, ganadería, silvicultura y pesca (1) & 0,951 & 1,011 & 0,972 & 0,995 \\
Construcción e instalaciones (2) & 0,951 & 1,062 & 1,206 & 0,974 \\
Servicios comunitarios, sociales y personales (3) & 0,966 & 1,011 & 1,059 & 1,342 \\
Electricidad, gas y agua (4) & 1,203 & 1,041 & 1,342 \\
Servicios financieros, de seguros, inmobiliarios y a las empresas (5) & 0,943 & 1,108 & 1,299 & 1,148 \\
Minería, canteras y refinado (6) & 0,695 & 0,879 & 1,363 & 1,247 \\
Manufacturas (7) & 0,874 & 1,037 & 1,134 & 1,026 \\
Transporte, almacenamiento y comunicaciones (8) & 0,909 & 1,028 & 1,229 & 1,007 \\
Mayorista, minorista, hoteles y restaurantes (9) & 0,827 & 0,999 & 1,101 & 0,949 \\
Todos los grupos de sectores & 0,927 & 1,030 & 1,172 & 1,000 \\
\hline
\end{tabular}

Fuente: elaboración propia sobre la base de datos de la Encuesta sobre la Fuerza de Trabajo, segundo trimestre de 2004 a 2007 , Instituto de Estadística de Jamaica (STATIN). 
de la media de esa ocupación. Si $\overline{r w_{k}}>1$, los salarios de los trabajadores en el sector $k$ son un $\left[\left(\overline{r w_{k}}-1\right) \mathrm{x}\right.$ 100]\% superiores a su promedio ocupacional.

Los datos contenidos en los cuadros 2 y 3 plantean la cuestión de si los porcentajes de la población activa de empleados en la categoría de empresas más pequeñas o en la de empresas de mayor tamaño explican la distribución sectorial de $\overline{r w_{k}}$. En todos los grupos de sectores, excepto en uno, $\overline{r w_{k}}$ es superior a uno para la categoría de empresas de mayor tamaño e inferior a uno para las más pequeñas (véase el cuadro 3). Además, la fuerza de trabajo de los sectores con $\overline{r w_{k}}>1$ suele estar más concentrada en las empresas de mayor tamaño y los trabajadores de los sectores con $\overline{r w_{k}}<1$ se concentran generalmente en empresas de menor tamaño (véanse los cuadros 2 y 3 ).

El cuadro 4 proporciona un significativo apoyo a esta hipótesis, ya que las mediciones de correlación paramétrica y no paramétrica indican la existencia de una marcada correlación positiva entre la concentración de la fuerza de trabajo en grandes empresas y los salarios relativos medios del sector $\left(\overline{r w_{k}}\right)$, a la vez que se encuentra una marcada correlación negativa entre la concentración de la fuerza de trabajo en pequeñas empresas y los salarios relativos promedio del sector.

El grado de correlación positiva (negativa) entre la concentración de la fuerza de trabajo en la categoría de empresas de mayor (menor) tamaño y el salario relativo medio de cada sector $\left(\overline{r w_{k}}\right)$ indica que existe una relación entre las desigualdades salariales según el tamaño de la empresa y las desigualdades salariales intersectoriales. Además, el hecho de que $\overline{r w_{k}}$ sea superior a uno (inferior a uno) para las grandes (pequeñas) empresas en todos los grupos de sectores, excepto en uno, muestra que, probablemente, la relación de causalidad va de las desigualdades salariales según el tamaño de la empresa a las desigualdades salariales intersectoriales y no en el sentido contrario.

CUADRO 4

\section{Coeficientes de correlación de Pearson y Spearman entre $\overline{r w_{k}}$ y la concentración de trabajadores en empresas de tamaños seleccionados}

\begin{tabular}{|c|c|c|c|c|c|c|}
\hline \multirow{2}{*}{ Grupo de sectores } & \multicolumn{2}{|c|}{$\begin{array}{l}\text { Proporción de trabajadores según } \\
\text { el tamaño de la empresa } \\
\text { (en porcentajes })\end{array}$} & \multirow{2}{*}{$\begin{array}{c}\text { Rango } \\
\text { (A) }\end{array}$} & \multirow{2}{*}{$\begin{array}{l}\text { Rango } \\
\text { (B) }\end{array}$} & \multirow{2}{*}{$\begin{array}{l}r w_{k} \\
\text { sector }\end{array}$} & \multirow{2}{*}{$\frac{\text { Rango }}{\left(\overline{r w_{k}}\right)}$} \\
\hline & $\begin{array}{l}50 \text { o más } \\
\text { trabajadores } \\
\text { (A) }\end{array}$ & $\begin{array}{l}10 \text { o menos } \\
\text { trabajadores } \\
\text { (B) }\end{array}$ & & & & \\
\hline Agricultura, ganadería, silvicultura y pesca (1) & 21,4 & 48,60 & 4 & 6 & 0,973 & 2 \\
\hline Construcción e instalaciones (2) & 5,2 & 67,16 & 2 & 8 & 0,995 & 4 \\
\hline Servicios comunitarios, sociales y personales (3) & 3,2 & 83,90 & 1 & 9 & 0,974 & 3 \\
\hline Electricidad, gas y agua (4) & 54,8 & 6,45 & 8 & 2 & 1,217 & 8 \\
\hline Servicios financieros, de seguros, inmobiliarios y a las empresas (5) & 34,9 & 16,46 & 7 & 3 & 1,148 & 7 \\
\hline Minería, canteras y refinado $(6)$ & 76,9 & 2,48 & 9 & 1 & 1,247 & 9 \\
\hline Manufacturas (7) & 32,0 & 25,96 & 6 & 4 & 1,026 & 6 \\
\hline Transporte, almacenamiento y comunicaciones (8) & 21,9 & 55,07 & 5 & 7 & 1,007 & 5 \\
\hline Mayorista, minorista, hoteles y restaurantes (9) & 18,3 & 39,96 & 3 & 5 & 0,949 & 1 \\
\hline Coeficiente de correlación de Pearson con $\overline{r w_{k}}$ & 0,908 & $-0,828$ & & & & \\
\hline Coeficiente de correlación de Spearman con $\overline{r w_{k}}$ & & & 0,867 & $-0,733$ & & \\
\hline
\end{tabular}

Fuente: elaboración propia sobre la base de datos de la Encuesta sobre la Fuerza de Trabajo, segundo trimestre de 2004 a 2007 , Instituto de Estadística de Jamaica (STATIN). 


\section{IV}

\section{Estimación y resultados: explicación de las desigualdades salariales según el tamaño de la empresa}

En el cuadro 3 se indica que, después de haber tenido en cuenta las características ocupacionales, los trabajadores de empresas con menos de 10 trabajadores reciben un salario por hora un $24,6 \%$ inferior, en promedio, al salario medio de los trabajadores de empresas con 50 empleados o menos. Este análisis resulta útil, pero su utilidad es limitada porque no tiene en cuenta otras características de los trabajadores, como la experiencia y los años de educación, y no explica el fenómeno de las desigualdades salariales según el tamaño de la empresa. Estas carencias pueden solucionarse, en cierta medida, examinando la magnitud y la significación estadística de los coeficientes $\alpha_{k}$ del tamaño de la empresa (ES) en la ecuación (1) del capital humano ampliada:

$$
\begin{gathered}
L A E=\alpha+\sum_{h=1}^{4} \alpha_{h} Y E A R_{h}+b_{2} H W K D+b_{3} H W K D^{2}+ \\
b_{4} E X P+b_{5} E X P^{2}+b_{6} Y S+b_{7} Y S^{2}+b_{8} T M A T \times Y S+ \\
b_{9} T M A T \times Y S^{2}+\alpha_{1} T M A T+\sum_{i=1}^{8} \alpha_{i} O C C_{i}+ \\
\sum_{i=1}^{8} \alpha_{i, 1} O C C_{i} O J T+\sum_{i=1}^{8} \alpha_{i, 2} O C C_{i} G E N+ \\
\sum_{j=1}^{8} \alpha_{j} I N D_{j}+\sum_{k=1}^{2} \alpha_{k} E S_{k}+\sum_{l=1}^{2} \alpha_{l} T E N_{l}+ \\
\sum_{m=1}^{2} \alpha_{m} K R U_{m}
\end{gathered}
$$

donde $\alpha$ es el parámetro principal de intersección; $a_{j}$ es el parámetro de desplazamiento-intersección de la variable ficticia número $j$ en el grupo asociado de variables ficticias $\forall h, i, j, m ; \mathrm{a}_{j l}$ es otro parámetro de desplazamiento resultante de la interacción de las variables ficticias números $j$ y $l$ en sus respectivos grupos ficticios $\forall j, l$; y $b_{i}$ es el parámetro de pendiente para su variable asociada, $\forall \mathrm{I}=1,2, \ldots, 9$.

En la ecuación (1) se incluyen diversas variables que proporcionan una indicación de la calidad del trabajador: la experiencia $(E X P)$, los años de educación $(Y S)$, si el trabajador se ha matriculado o podría haberse matriculado en una institución de estudios terciarios (TMAT) y si el trabajador ha recibido capacitación formal en el empleo (OJT).
Si se determinase que cada uno de los dos parámetros de desplazamiento-intersección del tamaño de la empresa son iguales a cero $\left(\alpha_{k}=0\right)$, entonces podría deducirse que las explicaciones basadas en la calidad (medida) de la mano de obra (LQE) pueden dar razón de las desigualdades salariales estimadas según el tamaño de la empresa (véase el cuadro 3). Sin embargo, si se determinase que, al menos en una ocasión, $\alpha_{k} \neq 0$ (una prima dependiente del tamaño del empleador o ESP), se estimará una modificación de la ecuación (1) para establecer en qué medida resulta apropiado incorporar las diferencias de tamaño de las empresas en los perfiles de permanencia en el empleo, como explicación auxiliar de las desigualdades salariales según el tamaño de la empresa. Esto supone incorporar una variable ficticia de la interacción entre el tamaño de la empresa y la permanencia en el empleo, es decir, añadir $\sum_{l=1}^{2} \sum_{k=1}^{2} \alpha_{l k} T E N_{l} E S_{k}$ a la ecuación (1).

Para todas las estimaciones, se utilizó el programa computacional estadístico STATA-12.1. Cuando se estima la ecuación (1) utilizando mínimos cuadrados ordinarios (MCO), la prueba para determinar la presencia de valores atípicos influyentes indica que hay 338 de estos valores en la serie de datos (de un total de 7.186), los que probablemente contribuyan a la fuerza explicativa relativamente baja del modelo (coeficiente de determinación o $\mathrm{R}^{2}$ de 0,397$)^{12}$. Es posible hacer dos cosas: i) utilizar una técnica de estimación robusta o resistente (en relación con los valores atípicos), o ii) eliminar algunas o todas las observaciones identificadas como valores atípicos influyentes. Esta segunda posibilidad es aconsejable si los valores atípicos son claramente espurios (Cousineau y Chartier, 2010; Osborne y Overbay, 2004). Si bien las técnicas de estimación robusta protegen en principio las estimaciones de parámetros contra las distorsiones provocadas por valores atípicos, sin los riesgos vinculados a la eliminación de datos atípicos, no otorgan el mismo peso a cada valor

12 Los valores atípicos influyentes se identificaron utilizando el método de la distancia de Cook para determinar la influencia, con el límite habitual (4/n). 
de la regresión, de manera que introducen un cierto grado de juicio de valor ${ }^{13}$.

Los resultados presentados en este estudio se basan en la muestra original de 7.186 participantes, menos $36 \mathrm{de}$ los 338 valores atípicos influyentes. A continuación, se explica brevemente el proceso de selección de estos 36 valores atípicos.

El gráfico 1 representa el logaritmo de los ingresos anuales brutos del empleo principal ( $L A E)$ y el $r w$ de los 338 valores atípicos influyentes del modelo ${ }^{14}$. En este

13 Véase en Verardi y Croux (2009) una descripción técnica de los diversos tipos de métodos de regresión robusta.

14 Las referencias superior e inferior de los valores atípicos de la distribución de $r w$ se determinaron aplicando primero a $r w$ la transformación de la raíz cuadrada modificada, tal como proponen Cousineau y Chartier (2010):

$$
r w^{\prime}=\sqrt{\frac{r w-r w_{l}}{r w_{h}-r w_{l}}}
$$

donde $r w_{h}$ y $r w_{l}$ son los valores más alto y más bajo de $r w$. Esta transformación convirtió la distribución sesgada hacia la derecha $(r w)$ en una distribución simétrica $\left(r w^{\prime}\right)$. Se utilizó un diagrama de caja tradicional para identificar límites estrictos de valores atípicos para la distribución $r w^{\prime}$. Posteriormente, se realizaron las transformaciones adecuadas para convertir estos límites de valores atípicos de $r w^{\prime}$ en límites de valores atípicos para la distribución $r w$ original. estudio, esos valores atípicos, cuando son identificados tanto por el modelo de regresión (valores atípicos influyentes) como por la distribución de $r w$, se denominan valores atípicos dobles. Ocho de los 36 valores atípicos dobles son valores atípicos bajos, mientras que 28 son valores atípicos altos.

Se consideró que los 36 valores atípicos dobles habían sido incluidos en los datos de forma ilegítima (Osborne y Overbay, 2004) y, por lo tanto, se eliminaron ${ }^{15}$. Los

${ }^{15}$ Los límites de valores atípicos para $r w$ son 0,004068 en el extremo inferior y 3,416260 en el extremo superior. Se denominan valores atípicos dobles aquellos valores atípicos que han sido identificados por el modelo como altamente influyentes y cuyo salario por hora o bien es inferior al $0,4 \%$ de su media ocupacional o superior al $242 \%$ de los ingresos medios de su código de ocupación de cuatro dígitos. En el extremo inferior, siete de las ocho personas indicaron que sus ingresos eran inferiores a tres dólares al año. En cuanto a los 28 valores atípicos dobles del extremo superior, la media salarial corresponde a 5,7 veces el salario medio de su grupo ocupacional de cuatro dígitos respectivo. Las personas del primer cuartil tenían un salario entre 3,5 y 3,6 veces superior al salario medio de su ocupación y el salario de los integrantes del último cuartil era más de 7,8 veces superior al promedio ocupacional (una persona afirmó que sus ingresos anuales eran de más de 21 millones de dólares, es decir, 23,8 veces el salario medio de su ocupación). Es probable que, en estos casos, se haya cometido un error (de forma deliberada o no) en los ingresos indicados o en el código ocupacional.

GRÁFICO 1

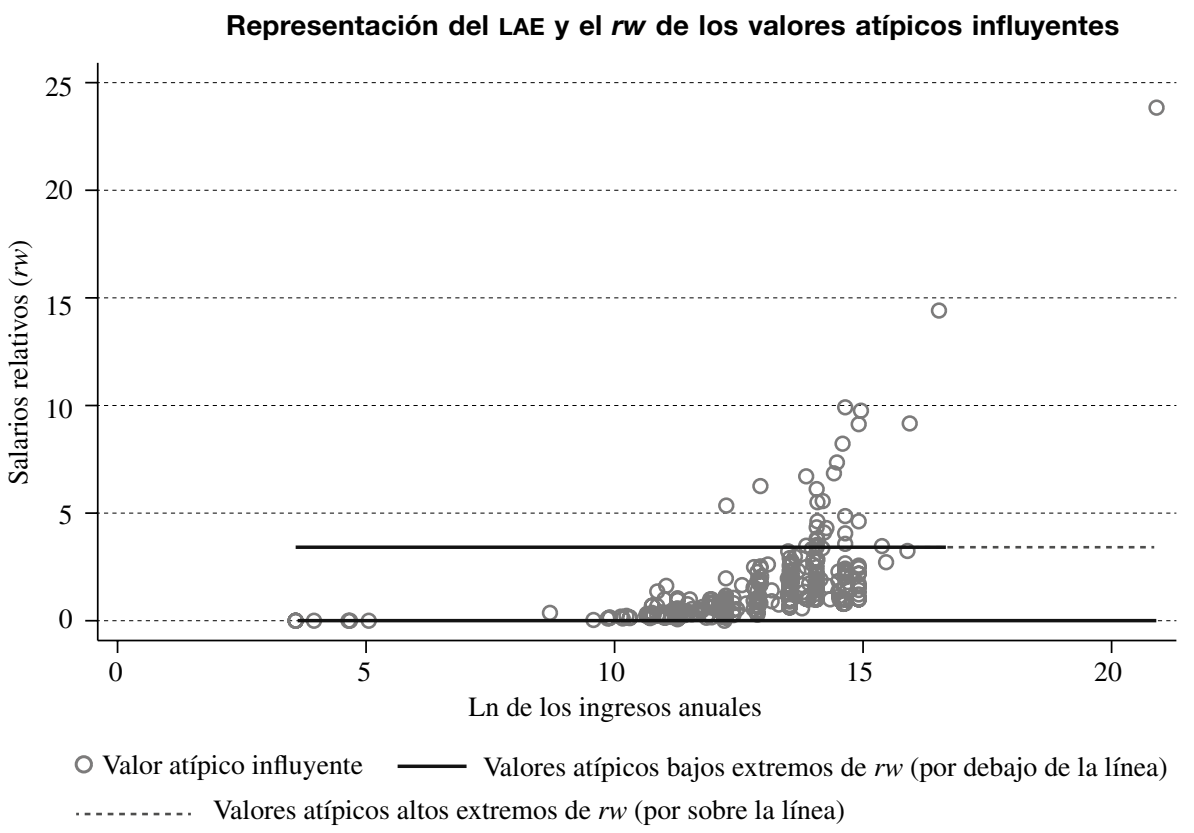

Fuente: elaboración propia sobre la base de datos de la Encuesta sobre la Fuerza de Trabajo, segundo trimestre de 2004 a 2007 , Instituto de Estadística de Jamaica (statin).

Nota: los puntos por debajo de la línea continua indican salarios relativos $(r w)$ inferiores al punto de referencia inferior de valores atípicos en la distribución $r w$; los puntos por sobre la línea discontinua representan salarios relativos $(r w)$ superiores al punto de referencia superior de valores atípicos de la distribución $r w$.

LAE: logaritmo de los ingresos anuales brutos del empleo principal. 
restantes 338 valores atípicos influyentes originales se conservaron en la serie de datos.

En el cuadro 5 se evidencian los resultados de la estimación de la ecuación (1). La estimación se basa en 7.150 respuestas a la encuesta, tras haber retirado los 36 valores atípicos dobles. El modelo explica alrededor del $46,8 \%$ de la variación en el logaritmo de los ingresos anuales. Al incluir los 36 valores atípicos dobles en la serie (7.186 datos), los resultados de las regresiones (que no se muestran en este estudio) fueron muy similares a los del cuadro 5, con un coeficiente de determinación $\left(\mathrm{R}^{2}\right)$ del 39,7\%. La regresión robusta (que no se muestra) también produce resultados similares.

Los coeficientes tienen en general el signo previsto. Los datos sobre experiencia dan lugar a una curva cóncava (aumentan a una velocidad cada vez menor), al igual que los datos sobre horas trabajadas. Las personas que han trabajado durante cinco años o más en su empresa actual ganan un $9,1 \%$ más que los trabajadores con una permanencia en el empleo de dos años o menos.

CUADRO 5

Producto de la estimación: ecuación (1)

\begin{tabular}{|c|c|c|c|c|c|c|}
\hline Variable explicativa & Coeficiente & Error estándar & Estadístico $t$ & $P>|t|$ & \multicolumn{2}{|c|}{ Intervalo de confianza del $95 \%$} \\
\hline \multicolumn{7}{|l|}{ AÑO } \\
\hline 2005 & 0,0906 & 0,0148 & 6,14 & 0,0000 & 0,0616 & 0,1195 \\
\hline 2006 & 0,1824 & 0,0143 & 12,74 & 0,0000 & 0,1543 & 0,2104 \\
\hline 2007 & 0,2343 & 0,0158 & 14,84 & 0,0000 & 0,2033 & 0,2652 \\
\hline \multicolumn{7}{|l|}{ IND } \\
\hline 2 & 0,3073 & 0,0325 & 9,45 & 0,0000 & 0,2435 & 0,3711 \\
\hline 4 & 0,5445 & 0,1054 & 5,17 & 0,0000 & 0,3380 & 0,7510 \\
\hline 5 & 0,2609 & 0,0363 & 7,19 & 0,0000 & 0,1898 & 0,3321 \\
\hline 6 & 0,6126 & 0,0544 & 11,26 & 0,0000 & 0,5060 & 0,7192 \\
\hline 7 & 0,1364 & 0,0341 & 4,00 & 0,0000 & 0,0696 & 0,2033 \\
\hline 8 & 0,1892 & 0,0374 & 5,06 & 0,0000 & 0,1160 & 0,2625 \\
\hline 9 & 0,0700 & 0,0303 & 2,31 & 0,0210 & 0,0105 & 0,1295 \\
\hline \multicolumn{7}{|l|}{$\mathrm{OCC}^{\mathrm{a}}$} \\
\hline 3 & 0,1373 & 0,0526 & 2,61 & 0,0090 & 0,0342 & 0,2404 \\
\hline 5 & 0,3066 & 0,0921 & 3,33 & 0,0010 & 0,1262 & 0,4871 \\
\hline 6 & $-0,1336$ & 0,0609 & $-2,19$ & 0,0280 & $-0,2529$ & $-0,0143$ \\
\hline 7 & 0,4889 & 0,1095 & 4,46 & 0,0000 & 0,2742 & 0,7037 \\
\hline 8 & $-0,2415$ & 0,1506 & $-1,60$ & 0,1090 & $-0,5368$ & 0,0537 \\
\hline 9 & $-0,0249$ & 0,0510 & $-0,49$ & 0,6250 & $-0,1248$ & 0,0750 \\
\hline 10 & 0,1150 & 0,0665 & 1,73 & 0,0840 & $-0,0153$ & 0,2453 \\
\hline HWKD & 0,0226 & 0,0051 & 4,41 & 0,0000 & 0,0126 & 0,0326 \\
\hline $\mathrm{HWKD}^{2}$ & $-0,0002$ & 0,0001 & $-3,55$ & 0,0000 & $-0,0003$ & $-0,0001$ \\
\hline \multicolumn{7}{|l|}{ ES } \\
\hline 10-49 empleados & 0,1432 & 0,0133 & 10,73 & 0,0000 & 0,1170 & 0,1693 \\
\hline$\geq 50$ empleados & 0,2285 & 0,0183 & 12,48 & 0,0000 & 0,1926 & 0,2643 \\
\hline EXP & 0,0119 & 0,0017 & 7,13 & 0,0000 & 0,0086 & 0,0151 \\
\hline $\mathrm{EXP}^{2}$ & $-0,0001$ & 0,0000 & $-5,08$ & 0,0000 & $-0,0002$ & $-0,0001$ \\
\hline TMAT & $-0,8870$ & 1,9454 & $-0,46$ & 0,6480 & $-4,7006$ & 2,9265 \\
\hline YS & 0,0343 & 0,0224 & 1,53 & 0,1260 & $-0,0097$ & 0,0783 \\
\hline TMAT\#YS ${ }^{2}$ & $-0,0053$ & 0,0112 & $-0,47$ & 0,6360 & $-0,0273$ & 0,0167 \\
\hline \multicolumn{7}{|l|}{ OCC\#OJT $^{b}$} \\
\hline 2\#1 & 0,0742 & 0,0289 & 2,57 & 0,0100 & 0,0176 & 0,1309 \\
\hline $3 \# 1$ & 0,1522 & 0,0343 & 4,44 & 0,0000 & 0,0850 & 0,2194 \\
\hline $4 \# 1$ & 0,1777 & 0,0576 & 3,08 & 0,0020 & 0,0647 & 0,2907 \\
\hline $5 \# 1$ & 0,3549 & 0,1063 & 3,34 & 0,0010 & 0,1464 & 0,5633 \\
\hline $6 \# 1$ & $-0,0127$ & 0,0643 & $-0,20$ & 0,8430 & $-0,1388$ & 0,1133 \\
\hline $7 \# 1$ & $-0,0137$ & 0,0993 & $-0,14$ & 0,8910 & $-0,2084$ & 0,1811 \\
\hline $8 \# 1$ & 0,2617 & 0,0843 & 3,11 & 0,0020 & 0,0965 & 0,4268 \\
\hline 9\#1 & 0,1057 & 0,0244 & 4,33 & 0,0000 & 0,0578 & 0,1537 \\
\hline $10 \# 1$ & 0,2370 & 0,0558 & 4,25 & 0,0000 & 0,1277 & 0,3463 \\
\hline
\end{tabular}




\begin{tabular}{|c|c|c|c|c|c|c|}
\hline Variable explicativa & Coeficiente & Error estándar & Estadístico $t$ & $P>|t|$ & Intervalo de & ianza del 95\% \\
\hline \multicolumn{7}{|l|}{ TEN } \\
\hline 2-5 años & 0,0269 & 0,0168 & 1,60 & 0,1100 & $-0,0061$ & 0,0599 \\
\hline$\geq 5$ años & 0,0909 & 0,0162 & 5,60 & 0,0000 & 0,0591 & 0,1227 \\
\hline \multicolumn{7}{|l|}{$\mathrm{KRU}^{\mathrm{c}}$} \\
\hline Rural & $-0,1673$ & 0,0132 & $-12,69$ & 0,0000 & $-0,1931$ & $-0,1414$ \\
\hline Urbano & $-0,1472$ & 0,0142 & $-10,34$ & 0,0000 & $-0,1752$ & $-0,1193$ \\
\hline \multicolumn{7}{|l|}{ OCC\#GEN $^{\text {b }}$} \\
\hline 2 \#1 & 0,2448 & 0,0496 & 4,94 & 0,0000 & 0,1476 & 0,3420 \\
\hline $3 \# 1$ & 0,1131 & 0,0380 & 2,98 & 0,0030 & 0,0387 & 0,1876 \\
\hline 4\# 1 & 0,0637 & 0,0227 & 2,81 & 0,0050 & 0,0193 & 0,1081 \\
\hline $5 \# 1$ & 0,0903 & 0,1024 & 0,88 & 0,3770 & $-0,1103$ & 0,2910 \\
\hline $6 \# 1$ & 0,4443 & 0,0474 & 9,37 & 0,0000 & 0,3514 & 0,5373 \\
\hline $7 \# 1$ & 0,0272 & 0,0750 & 0,36 & 0,7170 & $-0,1198$ & 0,1742 \\
\hline $8 \# 1$ & 0,2998 & 0,1470 & 2,04 & 0,0410 & 0,0116 & 0,5880 \\
\hline $9 \# 1$ & 0,1124 & 0,0214 & 5,24 & 0,0000 & 0,0704 & 0,1544 \\
\hline $10 \# 1$ & 0,1963 & 0,0571 & 3,43 & 0,0010 & 0,0842 & 0,3083 \\
\hline Constante & 10,8079 & 0,1639 & 65,96 & 0,0000 & 10,4867 & 11,1291 \\
\hline \multicolumn{7}{|l|}{ Resumen estadístico } \\
\hline $\mathrm{N}^{\mathrm{o}}$ de respuestas & 7,150 & & & & & \\
\hline$F(52,7097)$ & 99,04 & & & & & \\
\hline Prob $>F$ & 0,0000 & & & & & \\
\hline$R^{2}$ & 0,4675 & & & & & \\
\hline$R^{2}$ ajustado & 0,4636 & & & & & \\
\hline \multicolumn{6}{|l|}{ Raíz cuadrada del error } & \\
\hline \multicolumn{7}{|l|}{ Criterio de información de } \\
\hline Akaike & 8716,73 & & & & & \\
\hline
\end{tabular}

Fuente: elaboración propia sobre la base de datos de la Encuesta sobre la Fuerza de Trabajo, segundo trimestre de 2004 a 2007 , Instituto de Estadística de Jamaica (STATIN).

Nota: la variable dependiente es el logaritmo de los ingresos brutos anuales del trabajo principal del trabajador (LAE). Las variables explicativas son el año; el grupo de sectores principal (IND); el grupo ocupacional principal del trabajador (OCC); las horas regulares de trabajo a la semana (HWKD); el tamaño de la empresa, medido como el número de personas empleadas en el lugar principal de trabajo del trabajador (ES); los años de experiencia (EXP); si los logros educativos del trabajador le permiten matricularse en estudios terciarios (TMAT); los años de educación efectiva (YS); si el trabajador ha recibido capacitación formal en el empleo (OJT); permanencia o antigüedad en el trabajo actual (TEN); si el lugar de trabajo está situado en la capital, Kingston, en un área rural o en un área urbana (KRU), y variables ficticias de interacción.

a El primer grupo ocupacional importante, las fuerzas armadas, no tiene participantes en la encuesta del sector privado.

b El signo \# representa la interacción entre las variables concernidas.

c El área metropolitana de Kingston (KMA) es la categoría excluida.

\section{Evaluación de la validez de la explicación basada en la calidad de la mano de obra}

El cuadro 5 denota que, después de tener en cuenta el efecto del capital humano medido y de otras características de los trabajadores, ambos parámetros de desplazamientointersección del tamaño de la empresa son significativos y mayores que cero $\left(\alpha_{k}>0\right)$. Se estima que las empresas con entre 10 y 49 empleados y las empresas con 50 o más empleados pagan una prima (ESP) del 14,3\% y del 22,9\%, respectivamente, en comparación con los salarios pagados por empresas con menos de 10 empleados (categoría excluida). Las primas ESP previstas son similares a los diferenciales medios del 10,3\% (empresas con entre $10 \mathrm{y}$ 49 empleados) y del 24,6\% (empresas con 50 empleados o más) en el cuadro 3 , en el que las horas trabajadas y la ocupación (código de cuatro dígitos) fueron las únicas variables controladas.

Por lo tanto, tener en cuenta la calidad de la mano de obra no elimina las desigualdades salariales según el tamaño de la empresa. El origen de las desigualdades salariales según el tamaño de la empresa no puede explicarse mediante los datos observados de calidad de la mano de obra.

\section{Perfiles de permanencia en el empleo}

La aparente incapacidad de la explicación basada en la calidad de la mano de obra (LQE) para dar razón de las desigualdades salariales según el tamaño de la empresa 
requiere que se evalúen explicaciones alternativas. Una alternativa que puede comprobarse experimentalmente en el contexto de las variables incluidas en la Encuesta sobre la Fuerza de Trabajo es la diferencia en perfiles de permanencia en el empleo. Para evaluar la validez de la hipótesis de que las diferencias de los perfiles de permanencia en el empleo son la causa de la prima salarial (ESP) observada según el tamaño de la empresa, se modificó la ecuación (1) relacionando el tamaño del empleador $\left(E S_{k}\right)$ y la variable categorizada $\left(T E N_{l}\right)$, y después se volvieron a evaluar el tamaño y la relevancia del parámetro de desplazamiento-intersección $\alpha_{k}$ del tamaño de la empresa $\left(E S_{k}\right)$. En el gráfico 2 se aprecian los perfiles de permanencia en el empleo estimados para empresas con menos de 10 empleados, entre 10 y 49 empleados y 50 o más empleados ${ }^{16}$. El punto correspondiente al resultado previsto para el trabajador medio en cada una de las tres categorías de tamaño de las empresas se indica mediante las formas geométricas respectivas, y las cotas a ambos lados señalan la amplitud del intervalo de confianza del 95\%. Como se muestra en el gráfico 2, los posibles tamaños de las empresas tienen diferentes

${ }^{16}$ Los perfiles de permanencia en el empleo estimados se dedujeron de las estimaciones de la ecuación (1) modificada. perfiles estimados de permanencia en el empleo en los intervalos considerados, y el salario de los trabajadores aumenta a medida que se incrementa la permanencia en el empleo ${ }^{17}$. La diferencia de salarios (datos de la encuesta) entre una empresa con 50 trabajadores o más y una con menos de 10 trabajadores es menor en el nivel más bajo de permanencia en el empleo y aumenta en los niveles más altos de permanencia en este ${ }^{18}$. Esta observación es coherente, en cierta medida, con la tesis principal de la explicación de las desigualdades salariales según el tamaño de la empresa basada en los perfiles de permanencia en el empleo. Sin embargo, el hecho de que los datos observados para las pequeñas empresas en el nivel más bajo de permanencia en el empleo sean estadísticamente diferentes de los datos para las grandes empresas puede sugerir que los perfiles de dicha permanencia no explican completamente, por sí solos, la ESP.

\footnotetext{
17 Para cada categoría de tamaño de la empresa, las pruebas estadísticas indican (a un nivel del 2\%) que el resultado previsto para cinco años o menos es superior que el resultado para dos años o menos.

18 Las diferencias de intervalo entre los resultados previstos para las empresas con 50 empleados o más y para las empresas con menos de 10 empleados en el nivel más bajo de permanencia en el empleo son estadísticamente menores que el intervalo en otros niveles de permanencia en el empleo.
}

GRÁFICO 2

Perfiles de permanencia en el empleo según el tamaño de la empresa

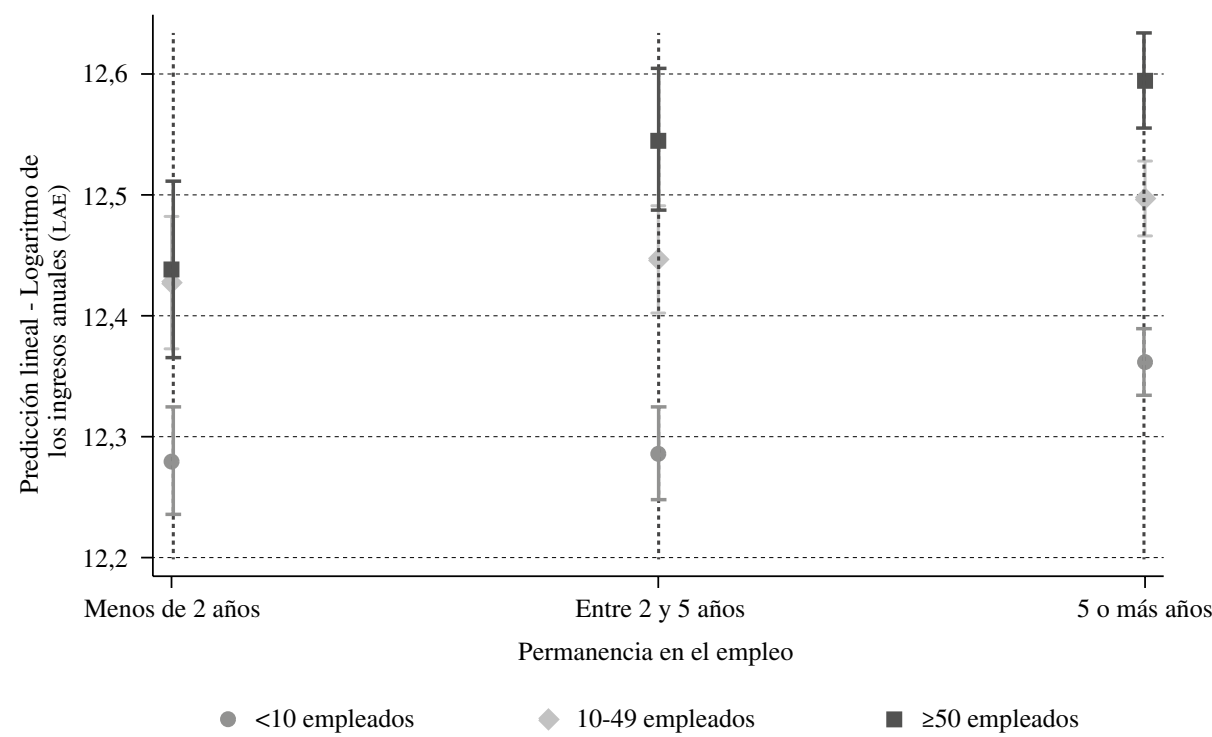

Fuente: elaboración propia sobre la base de datos de la Encuesta sobre la Fuerza de Trabajo, segundo trimestre de 2004 a 2007 , Instituto de Estadística de Jamaica (statin).

Nota: intervalos de confianza del $95 \%$. 
En el cuadro 6 se examina si la permanencia media en el empleo es diferente para distintos tamaños de las empresas. La antigüedad media en el empleo actual se calcula utilizando puntos medios en las categorías con límites y el límite inferior en el caso de la categoría ilimitada ${ }^{19}$. En el cuadro 6 se sugiere que los empleados de la categoría es de mayor tamaño y los de la categoría de menor tamaño permanecen durante más tiempo en sus empresas, en promedio, que los que trabajan para empresas con entre 10 y 49 empleados $^{20}$. Los perfiles de permanencia en el empleo observados en el gráfico 2 no son suficientes para explicar el fenómeno mostrado en el cuadro 6. ¿Por qué deberían los trabajadores permanecer más tiempo en promedio en las empresas que les pagan

19 Para este cálculo, se utilizó la variable original con siete categorías. ${ }^{20}$ Alrededor del $63 \%$ de la muestra corresponde a la última categoría de permanencia en el empleo (cinco años o más). La precisión del promedio de años de dicha permanencia podría verse afectada por la imposibilidad de determinar la distribución de un porcentaje tan grande de la muestra. menos (las pequeñas empresas) por sus años de servicio? La hipótesis basada en las condiciones laborales podría explicar esta anomalía.

Una vez que se ha mostrado que los perfiles de permanencia en el empleo son diferentes según el tamaño del empleador, ¿es posible que esas diferencias expliquen las primas dependientes del tamaño del empleador (ESP) estimadas anteriormente? En el cuadro 7 se presenta la estimación de $\alpha_{k}$ (el parámetro de desplazamientointersección asociado al tamaño de la empresa $k$ ) para el modelo aumentado de interacción entre tamaño de la empresa y permanencia en el empleo, así como la estimación en el modelo original. Si bien al tener en cuenta los perfiles de permanencia en el empleo se redujo la prima salarial de las empresas con 50 empleados o más de aproximadamente el 22,8\% al 15,9\%, no disminuyó la prima pagada a los trabajadores en empresas con entre 10 y 49 empleados.

Los resultados relativos a la educación, la experiencia y las horas trabajadas no cambiaron de forma cualitativa en comparación con los del modelo original.

CUADRO 6

Promedio de antigüedad en el empleo actual, por tamaño de la empresa

\begin{tabular}{lcccccc}
\hline \multirow{2}{*}{ Tamaño de la empresa } & \multicolumn{2}{c}{$\begin{array}{c}\text { Promedio de antigüedad } \\
\text { en el empleo actual }\end{array}$} & & & \multicolumn{2}{c}{ Estadísticamente diferente de (valor $p$ unilateral) } \\
\cline { 2 - 3 } \cline { 5 - 7 } & Promedio & Error estándar & & $<10$ empleados & $10-49$ empleados & $\geq 50$ empleados \\
\hline$<10$ empleados & 4,159204 & 0,0213201 & & & 0,0130 & 0,2951 \\
$10-49$ empleados & 4,079413 & 0,0290760 & & 0,0130 & 0,1237 \\
$\geq 50$ empleados & 4,135558 & 0,0386143 & & 0,2951 & 0,1237 & \\
\hline
\end{tabular}

Fuente: elaboración propia sobre la base de datos de la Encuesta sobre la Fuerza de Trabajo, segundo trimestre de 2004 a 2007 , Instituto de Estadística de Jamaica (sTATIN).

CUADRO 7

Efecto de la interacción del tamaño de la empresa y la permanencia en el empleo en las desigualdades salariales, según el tamaño de la empresa

\begin{tabular}{lccc}
\hline Tamaño de la empresa $\left(E S_{k}\right)$ & $\begin{array}{c}\text { Modelo II } \\
\left(\alpha_{k}\right)\end{array}$ & $\begin{array}{c}\text { Modelo I } \\
\text { Original }\left(\alpha_{k}\right)\end{array}$ & $\begin{array}{c}\text { H0: II = I } \\
\text { (valor } p \text { unilateral) }\end{array}$ \\
\hline Entre 10 y 49 empleados & $0,1479^{\mathrm{a}}$ & $0,1432^{\mathrm{a}}$ & 0,8735 \\
50 o más empleados & $0,1588^{\mathrm{a}}$ & $0,2285^{\mathrm{a}}$ & 0,0690 \\
Resumen estadístico & & & \\
$\mathrm{R}^{2}$ & 0,4679 & 0,4675 & 0,4636 \\
$\mathrm{R}^{2}$ ajustado & 0,4637 & 8716,73 & \\
Criterio de información de Akaike & 8719,68 & & \\
\hline
\end{tabular}

Fuente: elaboración propia sobre la base de datos de la Encuesta sobre la Fuerza de Trabajo, segundo trimestre de 2004 a 2007 , Instituto de Estadística de Jamaica (statin).

a Estadísticamente significativo a un nivel del $1 \%$ o inferior. 


\section{V}

\section{Conclusiones}

La correlación positiva entre los salarios y el tamaño de la empresa, y sus consecuencias para la teoría del mercado laboral y la teoría de la empresa, adquieren una relevancia práctica adicional en el contexto de los altos niveles de desempleo y de una distribución de ingresos marcadamente sesgada que caracteriza a Jamaica y a gran parte del Caribe. En este estudio, se estima que -después de tener en cuenta el capital humano medido y otras características - los trabajadores de empresas con entre 10 y 49 empleados y de las empresas con más de 50 empleados reciben primas salariales del $14,3 \%$ y del $22,8 \%$, respectivamente, en comparación con los trabajadores de empresas con menos de 10 empleados.

En el estudio también se indica que la remuneración media de los trabajadores puede experimentar diferencias de hasta el 29,8\% en los distintos grupos de sectores (nivel de un dígito). Sin embargo, las correlaciones positivas (negativas) entre las categorías de empresas de mayor (menor) tamaño y la prima salarial del sector muestran que las desigualdades salariales, según el tamaño de la empresa, podrían ser la causa de las desigualdades salariales intersectoriales en Jamaica.

Aparentemente, los datos disponibles indican que las primas dependientes del tamaño del empleador (ESP) persisten incluso después de tener en cuenta los aspectos medidos de la calidad de la mano de obra. Por otra parte, las diferencias en los perfiles de permanencia en el empleo según el tamaño de las empresas parecen proporcionar una explicación parcial de la EsP estimada en Jamaica. Después de considerar esas diferencias, la ESP asociada a las empresas de mayor tamaño se reduce al $15,9 \%$, mientras que no hay reducción en la prima pagada por las empresas de tamaño mediano. No hay ninguna diferencia estadística en el promedio de antigüedad en el empleo entre las categorías de mayor y menor tamaño. Este fenómeno, junto con la magnitud de las primas no explicadas, abre la puerta a otras explicaciones teóricas de las desigualdades salariales según el tamaño de la empresa, como las basadas en las condiciones laborales, la ausencia de sindicatos o la eficiencia salarial, ninguna de las cuales se puede verificar con los datos disponibles actualmente.

Además de la limitación de los datos mencionada, la forma en que se miden las variables existentes en la serie de datos de la Encuesta sobre la Fuerza de Trabajo puede afectar al estudio de las desigualdades salariales según el tamaño de la empresa. La variable de permanencia en el empleo (TEN), por ejemplo, se mide de forma categorizada en la mencionada encuesta, de manera que más del $60 \%$ de los trabajadores del sector privado pertenecen a la última categoría (es decir, a una permanencia en el empleo de cinco años o más), lo que limita la variabilidad que puede determinarse. En consecuencia, un estudio más profundo de la hipótesis del perfil de permanencia en el empleo requeriría medidas más continuas de dicha permanencia de los trabajadores, en particular un desglose de lo que actualmente se engloba en la última categoría.

Finalmente, el efecto de las desigualdades salariales según el tamaño de la empresa en la distribución del ingreso constituye un gran incentivo para continuar investigándolas. Además, el estudio de esas desigualdades resulta fundamental para comprender el funcionamiento de los mercados laborales. Las causas que determinan las decisiones relativas a los salarios y al empleo influyen en el tipo de intervenciones en el mercado laboral que deberían planificarse para afrontar los niveles persistentemente altos de desempleo que caracterizan a Jamaica y a gran parte del Caribe. 


\section{Bibliografía}

Akerlof, G.A. (1984), "Gift exchange and efficiency-wage theory: four views", American Economic Review, vol. 74, $\mathrm{N}^{\circ} 2$, Nashville, Tennessee, American Economic Association.

(1982), "Labour contracts as a partial gift exchange", Quarterly Journal of Economics, vol. 97, º 4, Oxford, Oxford University Press.

Antos, J.R. (1983), "Union effects on white-collar compensation", Industrial and Labor Relations Review, vol. 36, $\mathrm{N}^{\circ} 3$, Cornell University

Arbache, J.S. (2001), "Wage differentials in Brazil: theory and evidence", Journal of Development Studies, vol. 38, $\mathrm{N}^{\circ} 2$, Taylor \& Francis.

Brown, C. y J. Medoff (1989), "The employer size-wage effect", Journal of Political Economy, vol. 97, $\mathrm{N}^{\circ}$ 5, Chicago, The University of Chicago Press.

Bulow, J.I. y L.H. Summers (1986), "A theory of dual labor markets with application to industrial policy, discrimination, and Keynesian unemployment", Journal of Labor Economics, vol. 4, $\mathrm{N}^{\circ}$ 3, Chicago, The University of Chicago Press.

Cousineau, D. y S. Chartier (2010), "Outliers detection and treatment: a review", International Journal of Psychological Research, vol. 3, No 1, Medellín, Universidad de San Buenaventura.

Duncan, G.J. y F.P. Stafford (1980), "Do union members receive compensating wage differentials?", American Economic Review, vol. 70, $\mathrm{N}^{\circ} 3$, Nashville, Tennessee, American Economic Association.

Fakhfakh, F. y F. Fitzroy (2006), "Dynamic monopsony: evidence from a French establishment panel", Economica, vol. 73, $\mathrm{N}^{\circ}$ 291, Londres, London School of Economics and Political Science.

Filer, R.K. (1985), "Male-female wage differences: the importance of compensating differentials", Industrial and Labor Relations Review, vol. 38, $\mathrm{N}^{\circ}$ 3, Cornell University.

Gittleman, M. y E.N. Wolff (1993), "International comparisons of inter-industry wage differentials", Review of Income and Wealth, vol. 39, $\mathrm{N}^{\circ} 3$, Wiley.

Hamermesh, D.S. y J. Grant (1979), "Econometric studies of laborsubstitution and their implications for policy", Journal of Human Resources, vol. 14, $\mathrm{N}^{\circ}$ 4, Wisconsin, University of Wisconsin Press.

Krueger, A.A. y L.H. Summers (1987), "Reflections on the interindustry wage structure", Unemployment and the Structure of Labor Markets, K. Lang y J. Leonard (eds.), Oxford, Basil Blackwell.

Lazear, E.P. (1981), "Agency, earnings profiles, productivity, and hours restrictions", American Economic Review, vol. 71, № 4 , Nashville, Tennessee, American Economic Association. (1979), "Why is there mandatory retirement?", Journal of Political Economy, vol. 87, $\mathrm{N}^{\circ}$ 6, Chicago, University of Chicago Press.

Lester, R. (1967), "Pay differentials by size of establishment", Industrial Relations, vol. 7, $\mathrm{N}^{\circ} 1$, Wiley.

Main, B.G.M. y B. Reilly (1993), "The employer size-wage gap: evidence for Britain", Economica, vol. 60, № 238, Wiley.
Masters, S.H. (1969), "An interindustry analysis of wages and plant size", Review of Economics and Statistics, vol. 51, $\mathrm{N}^{\circ} 3$, Cambridge, Massachusetts, The MIT Press.

Mellow, W. (1982), "Employer size and wages", Review of Economics and Statistics, vol. 64, $\mathrm{N}^{\circ} 3$, Cambridge, Massachusetts, The MIT Press.

Mounsey, A. y T. Polius (2011), “Trinidad y Tabago: Desigualdades salariales intersectoriales", Revista CEPAL, $\mathrm{N}^{\circ} 105$ (LC/G.2508-P), Santiago de Chile.

Oi, W. (1983), "The fixed employment costs of specialized labour", The Measurement of Labour Cost, J.E. Triplett (ed.), Chicago, University of Chicago Press.

Oosterbeek, H. y M. van Praag (1995), "Firm-size wage differentials in the Netherlands", Small Business Economics, vol. 7, $\mathrm{N}^{\circ} 3$, Springer.

Osborne, J.W. y A. Overbay (2004), "The power of outliers (and why researchers should always check for them)", Practical Assessment, Research \& Evaluation, vol. 9, $\mathrm{N}^{\circ} 6$.

Pearce, J.E. (1990), "Tenure, unions, and the relationship between employer size and wages", Journal of Labor Economics, vol. 8, $\mathrm{N}^{\circ} 2$, Chicago, The University of Chicago Press.

Riveros, L.A. y L. Bouton (1994), "Common elements of efficiency wage theories: what relevance for developing countries", Journal of Development Studies, vol. 30, $\mathrm{N}^{\circ} 3$, Taylor \& Francis.

Rosen, S. (1982), "Authority, control, and the distribution of earnings", Bell Journal of Economics, vol. 13, $\mathrm{N}^{\circ} 2$, Santa Monica, California, RAND Corporation.

Salop, S.C. (1979), "A model of the natural rate of unemployment", American Economic Review, vol. 69, № 1, Nashville, Tennessee, American Economic Association.

Schmidt, C. y K.F. Zimmermann (1991), "Work characteristics, firm size and wages", Review of Economics and Statistics, vol. 73, $\mathrm{N}^{\circ} 4$, Cambridge, Massachusetts, The MIT Press.

Shapiro, C. y J.E. Stiglitz (1984), "Equilibrium unemployment as a worker discipline device", American Economic Review, vol. 74, $\mathrm{N}^{\circ}$ 3, Nashville, Tennessee, American Economic Association.

Slichter, S. (1950), "Notes on the structure of wages", Review of Economics and Statistics, vol. 32, $\mathrm{N}^{\circ} 1$, Cambridge, Massachusetts, The MIT Press.

Smith, A. (1904), An Inquiry into the Nature and Causes of the Wealth of Nations, Londres, Methuen \& Co. [en línea] http:// www.econlib.org/library/Smith/smWN4.html.

Tan, H. y G. Batra (1997), "Technology and firm size-wage differentials in Colombia, Mexico, and Taiwan (China)", World Bank Economic Review, vol. 11, ํㅜ 1, Oxford, Oxford University Press.

Verardi, V. y C. Croux (2009), "Robust regression in Stata", The Stata Journal, vol. $9, \mathrm{~N}^{\circ} 3$.

Winter-Ebmer, R. y J. Zweimüller (1999), "Firm-size wage differentials in Switzerland: evidence from job-changers", American Economic Review, vol. 89, $\mathrm{N}^{\circ}$ 2, Nashville, Tennessee, American Economic Association.

Zwick, T. (2009), "Why pay seniority wages?", ZEW Discussion Paper, $\mathrm{N}^{\circ}$ 09-005, Mannheim, Centre for European Economic Research. 